\title{
SESSION 4
}

\section{Rotation and Stellar Evolution}




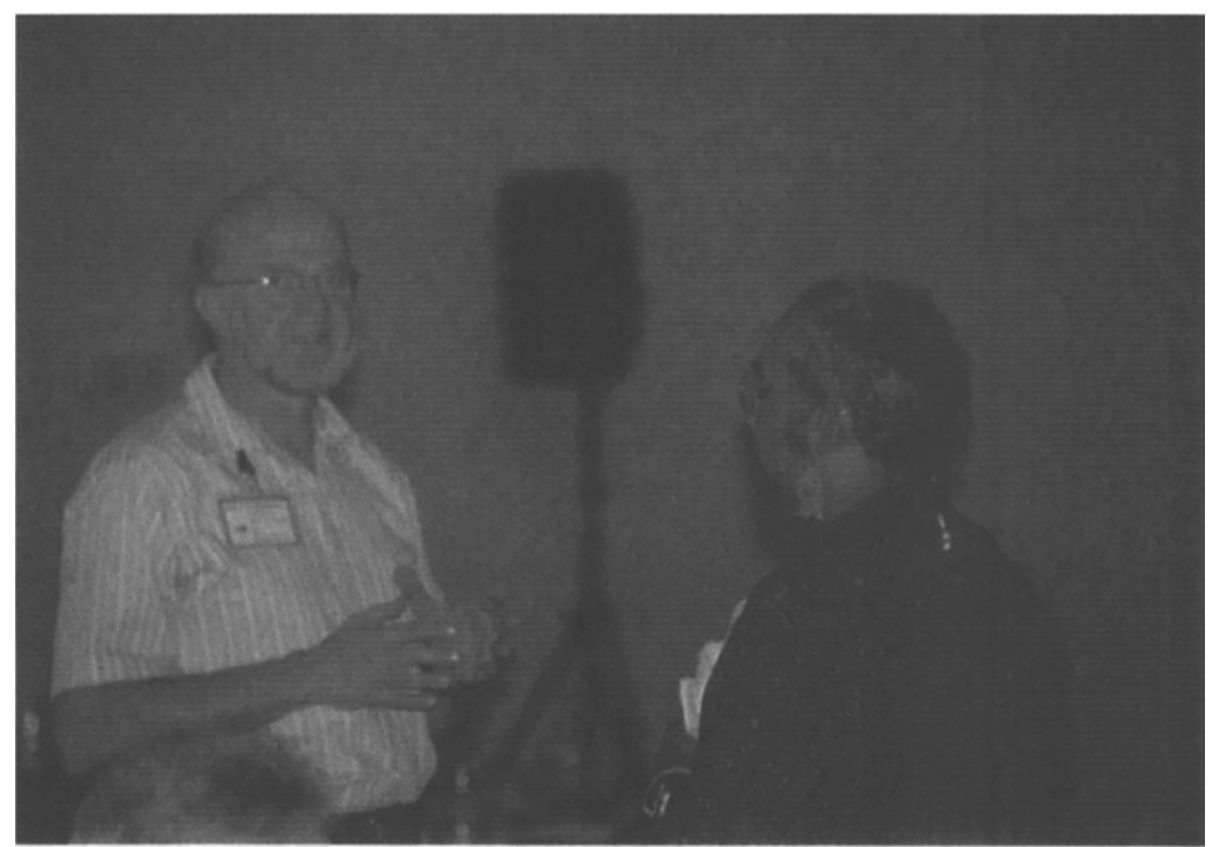

Juri Toomre and Gibor Basri in clarifying some matte.

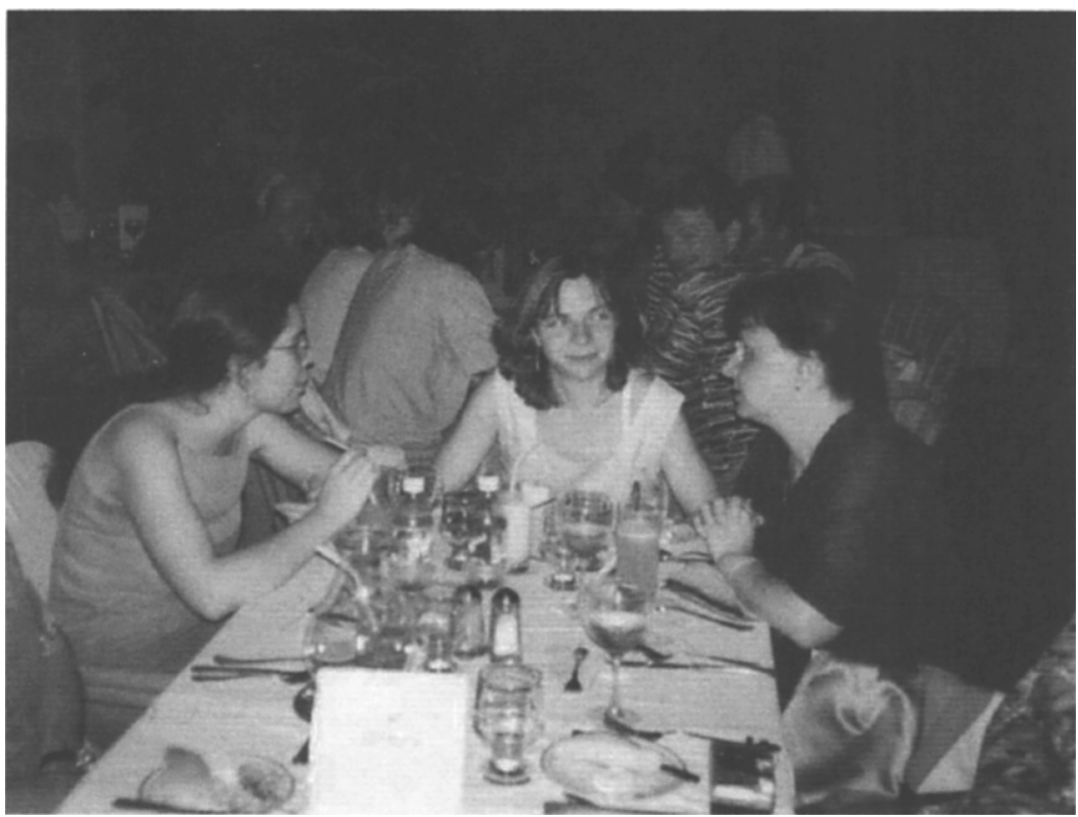

Ana Palacios, Suzanne Talon and Corinne Charbonnel discussing about horizontal turbulence. Frédéric Royer smiling in the background. 


\title{
Pre-Main Sequence Evolution of Rotating Stars in Relation with Observations of Young Clusters
}

\author{
Sabatino Sofia \\ Yale University, Dept. of Astronomy, Box 208101, New Haven, CT \\ 06520, USA \\ Sydney A. Barnes \\ Univ. of Wisconsin-Madison, Dept. of Astronomy, 475 N. Charter St., \\ Madison, WI 53703, USA and \\ Yale University, Dept. of Astronomy, Box 208101, New Haven, CT \\ 06520, USA
}

\begin{abstract}
Assuming that pre-main sequence stars rotating at the deuteriumburning birthline as solid bodies with rates within the range observed in T-Tauri stars is an adequate initial condition to explain the rotational properties of stars in clusters of all ages, provided that appropriate evolutionary schemes are used. It is, in fact, on the evolution that most recent advances have been made, and a new paradigm has arisen. Specifically, stellar periods (as a function of mass) begin their evolution on one of two branches, and the fast rotators migrate to the slow branch through a short-lived gap. These branches represent different physical properties of the internal stellar dynamics, and are related to the type of dynamo mechanism which operates within the stars. This paradigm appears to have the potential to address several fundamental issues in stellar astronomy.
\end{abstract}

Modeling stellar evolution is an initial value problem. We must start out with a model whose properties we presume to know, and advance it in time on the basis of the rates of change. Of course, establishing this initial model is not always straight-forward, and this is the subject of this paper.

For non-rotating stars, the ZAMS is an appropriate starting point. Excepting for some trace elements, the chemical composition is uniform at this stage, and subsequent evolution begins here. However, this is not the situation for rotating stars, since for a large mass range, by the time the ZAMS stage has been reached, two well defined (one radiative and one convective) zones have developed, and some form of differential rotation between them may have already been established.

The early studies of the evolution of rotating stars were made at a time when the observational information was very scant. For example, the best choice available to Endal \& Sofia (1981) was to start calculations also at the ZAMS stage, and assume that they rotated as solid bodies. Because the Kraft (1967) curve was already known, as it was the fact that the break in the curve occurred at the mass where outer convective zones develop, the starting velocities were obtained directly from the Kraft curve for high mass stars, and from the extrapolation of the high mass portion of the curve for low mass stars. The latter choice was justified by the reasonable assumption that the low mass stars, which possess magnetized stellar winds, had undergone magnetic torquing which had slowed 
down the rotation of the convective envelope to the low values usually observed for old late type stars.

Over the last two decades, the observational foundation of these studies has undergone an explosive growth, both by improvements in the techniques and by the extensive surveys carried out. This has allowed more sophisticated approaches to the problem, whose results we will review subsequently.

For low mass stars, there is a long-lived pre-MS phase where the entire star is convective. Although it is not possible from first principles to know what rotation law is asymptotically enforced in convective regions, (one could justify all choices from constant specific angular momentum to constant angular velocity), we can reasonably presume that such an asymptotic state is reached relatively quickly even for a rapidly changing pre-MS star. Palla \& Stahler (1991) made the suggestion that the appropriate starting point (or birthline) be associated with the deuterium-burning phase. Because this phase slows down evolution, (most observed T-Tauri stars occupy it), there should be enough time for the asymptotic rotation state to be reached. Also, although the helioseismologically determined rotation of the solar convective envelope is not exactly solid body, it is more closely solid-body than any other rotation law suggested. Thus, we should start out our calculations at the deuterium burning stage, assuming solid body rotation, and using the surface rotation rates observed in T-Tauri stars.

As an aside, let us address what set of the large amount of observational data should be considered in our study. The most relevant information is the angular velocity, $\omega$. The most abundant data currently available is $v \sin i$. We do not use these data in our analysis, however, because of the largest uncertainty of the inclination of the axis of rotation, and to a lesser extent, because of the uncertainties of the stellar radii, which link $v$ to $\omega$. Instead, we use rotational periods. Most low mass stars vary as a consequence of large star-spots, and it is possible to determine rotational periods with great accuracy, sometimes to 1 part in $10^{4}$. These are directly linked to angular velocities.

The surface rotation rate of T-Tauri stars, then, represent the range of rotation rates for stars at the birthline, and they are assumed to be rotating as solid bodies. Because of the trivial relationship we will refer to rotational periods, and rotational velocities, interchangeably.

Using the Yale Rotating Evolution Code (YREC), and assuming the wind torquing law proposed, for example, by Kawaler (1988), we can derive the rotation rates of all stars, as a function of radius, and time. For the purpose of comparison with observation, the surface velocities are particularly relevant. The T-Tauri rotational periods are mostly found to lie between 2 and 10 days, with extreme cases found between 0.5 and 16 days (Choi \& Herbst 1996; Stassun et al. 1999; Rebull 2001).

Earlier work, e.g. Pinsonneault et al. (1989), had shown that because of the feedbacks of the braking mechanism, all rotation rates quickly converged to similar values. The inclusion of pre-main sequence evolution and the correct range of starting periods does not change this convergent behavior (see, for instance Fig. 4 in Barnes \& Sofia 1996).

However, in most clusters with ages between tens and hundreds of million of years, we still found a range of rotation periods not much smaller than the one found in T-Tauri stars. In particular, young open clusters like the Pleiades (Van 


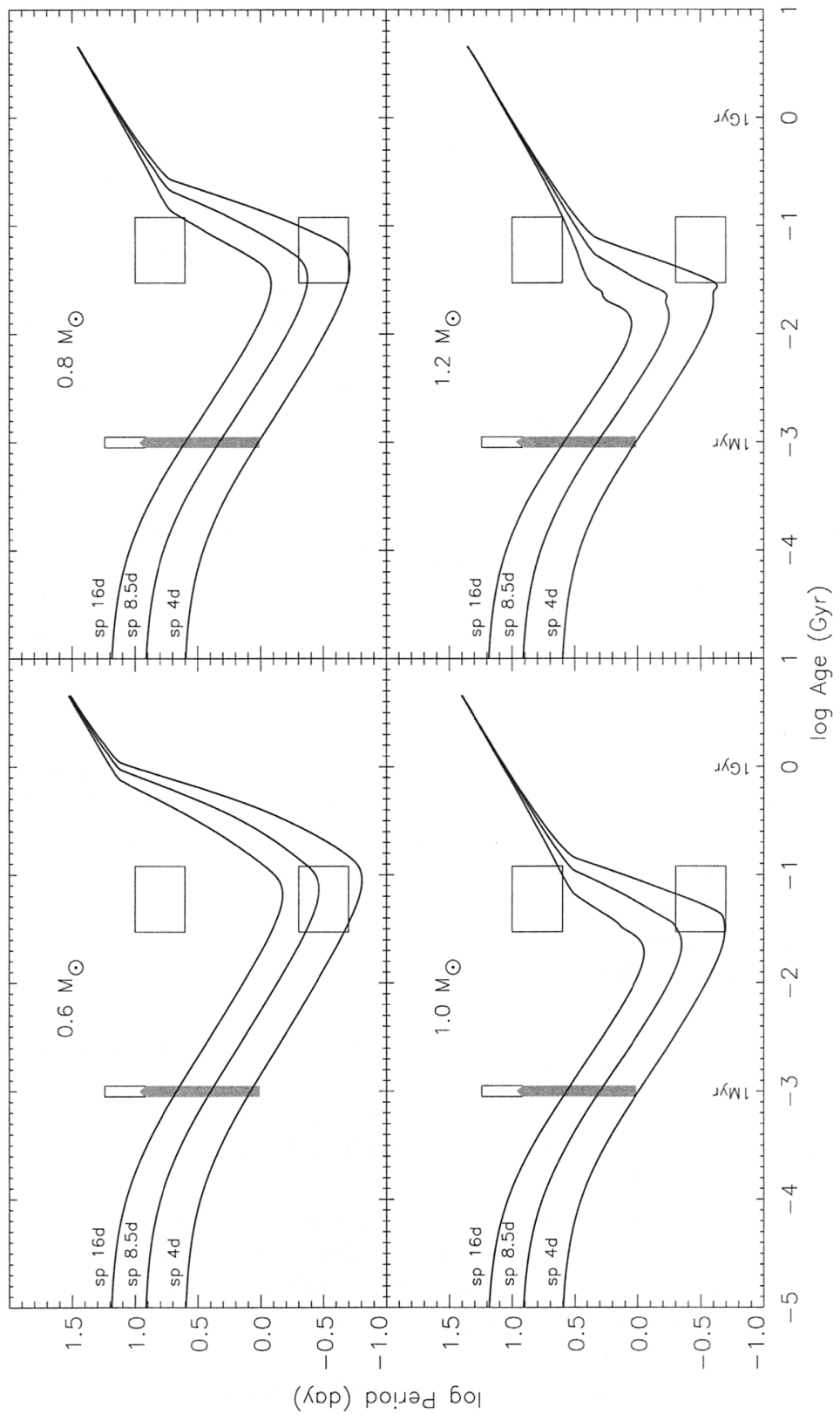

Figure 1. Magnetic saturation makes it possible to create the fast rotators observed in open clusters. Source: Barnes \& Sofia (1996). 
Leeuwen \& Alphenaar 1982; Van Leeuwen et al. 1987) were found to contain solar-type stars with periods as short as a fifth of a day. Similar objects were discovered subsequently in other young clusters, and these became known as ultra-fast rotators (UFRs).

In order to account for these ultra-fast rotators, we had to consider a massdependent saturation of the magnetic braking mechanism. MacGregor \& Brenner (1991) had suggested a kind of magnetic saturation, observational justification for saturation came from Stauffer (1994) in relation to X-ray data, and Chaboyer et al. (1995) implemented it in stellar models in an effort to understand the fast rotators, although pre-main sequence evolution was not included. The pre-main sequence became important when large numbers of rotation periods for T Tauri stars became available from work by Herbst and collaborators, and others later. However, including the pre-main sequence but no saturation does not help, as Fig. 4 in Barnes \& Sofia (1996) shows. So we were forced to consider saturation. And, in fact, it is possible to account for the UFRs in open clusters using magnetic saturation (Fig. 1).

However, this could not account for the simultaneous existence of slow rotators in these clusters. If you can model the fast rotators using magnetic saturation, you cannot at the same time have no saturation. The slowest of the saturated models rotates too fast compared to open cluster observations of slow rotators, and one is forced to consider an additional mechanism to account for the slow rotators.

Here, a scheme proposed originally by Edwards et al. (1993) can help. Based on their observational conclusions that T Tauri stars with infra-red excesses had longer rotation periods than those without, they proposed that magnetic interaction between a star and its disk might cause some stars to lose angular momentum rapidly. This offered a way to account for the fast rotators using magnetic saturation and simultaneously, the slow rotators using disk-interaction for some stars. The latter ones required the existence of circumstellar disks of the appropriate lifetimes (up to a few million years) magnetically linked to the convective envelope of the proto- star. We find disk life-times needed are of the order of 1-3 Myr (Fig. 2). Similar results were obtained by Krishnamurthi et al. (1997) and various others, although some studies suggested much longer timescales.

Thus by using both magnetic saturation and disk-interaction, it is possible to understand the main features of the rotational observations available. The overall idea is summarized in Fig. 3.

One can then try to figure out the mixture of stars of one kind or the other or the suite of parameters that would give the best fit to all the rotation observations available, and several studies attempted to do so. See Keppens et al. (1995) for an early attempt. For more recent work, see Tinker et al. (2002) and references therein.

Although the foundation of these explanations was reasonable, the fact that each time you got better observations it was necessary to specify the properties of the evolutionary processes became disturbing. It started to remind us of epicycles. Thus, it encouraged us to look anew at the problem, this time using the large database as a whole. 


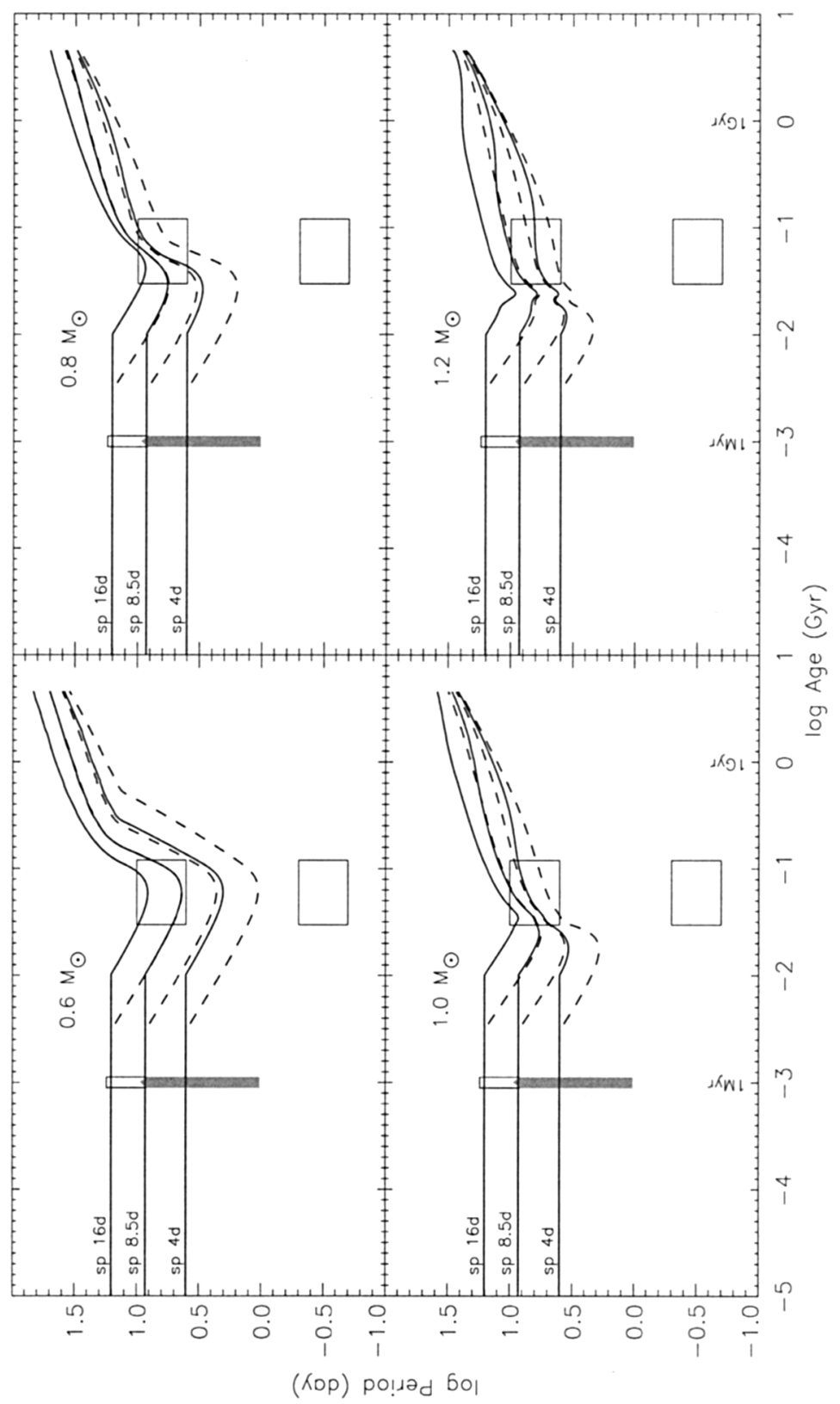

Figure 2. Disk interaction on the pre-main sequence makes it possible to explain the existence of the slow rotators observed in star clusters. The starting period in days is indicated by 'sp.' Source: Barnes, Sofia \& Pinsonneault (2001). 


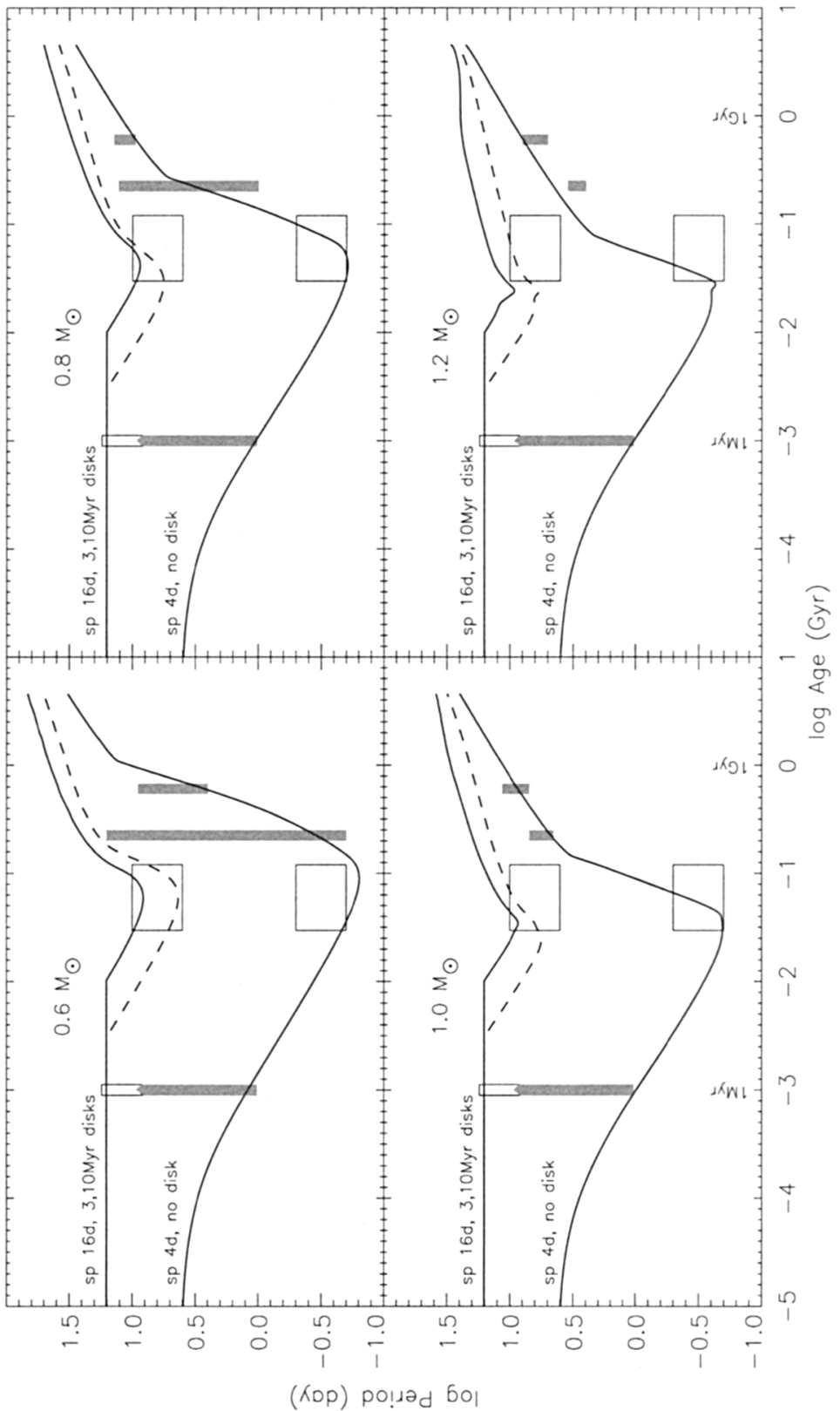

Figure 3. A combination of magnetic saturation and disk interaction can explain both the fast and slow rotators observed in young clusters. Source: Barnes, Sofia \& Pinsonneault (2001). 
Fig. 4, taken from Barnes (2003), displays this database. In addition to the open cluster observations, displayed in age-increasing order in the first six panels, it contains two panels displaying the Mt. Wilson stars (Wilson 1963), binned into two groups, young and old, as originally suggested by Vaughan (1980). In retrospect, the omission of these stars from rotational considerations was a serious shortcoming, understandable because of the difficulty of deciding their ages, but as shown in Barnes (2001), this can be alleviated considerably by using chromospheric ages provided by Soderblom et al. (1991) or Donahue (1998).

The open cluster observations appear not to comprise a single population but lie on two distinct sequences, with some stars in the rotational gap between them. The fraction of stars on each sequence changes systematically with stellar/cluster age, with stars migrating steadily from the fast sequence to the slow one.

The location of these sequences, represented by solid and dashed curves in Fig. 4, also changes systematically with stellar age. We find that we can describe the progressive evolution of the slow sequence by the product of $\sqrt{A g e}$, as suggested by the Skumanich (1972) relationship, and a universal function of stellar color/mass. This function of stellar mass turns out to be suspiciously close to $I^{-0.5}$, where $I$ is the moment of inertia of the entire star.

We find that we can describe the progressive evolution of the fast sequence by an exponential in stellar age that increases on a timescale that scales with the moment of inertia of only the outer convection zone. This suggests that only the outer convection zone participates in the initial spindown on the fast sequence, but the whole star participates in the spindown on the slow sequence.

A complete discussion of this appears in Barnes (2003), where it is suggested that this behavior may be understood in magnetic terms. Initially, only a smallscale magnetic field originating in convection is present, which cannot couple the convective zone to the inner radiative zone. Thus, there is a shear between them that creates a large-scale field via an interface-type dynamo, which then connects the two zones. This drives a star from the fast sequence through the gap onto the slow sequence, one occupied by the present-day Sun and the Mt. Wilson stars.

This scheme offers a comprehensive and predictive paradigm for interpreting the rotation rates of all solar- and late-type stars, including field stars, and may even allow us to derive rotational ages for stars. Future work will decide whether or not this new paradigm is valid.

\section{Concluding Remarks}

The principal conclusion of this paper is that the initial conditions from which to start the evolution of rotating (low mass) stars are relatively well defined. Beginning the calculations at the deuterium-burning birthline, with PMS stars rotating as solid bodies, and with rotational periods provided by observations of T-Tauri stars (mostly between 2 and 10 days, and with extreme cases between 0.5 and 16 days) is adequate to explain all subsequente evolution, i.e., observations of rotation rates of stars in open clusters of various ages, and the field Mt. Wilson stars.

Another important conclusion of this paper is that the most challenging aspect of the problem is actually to understand the mechanisms that govern 


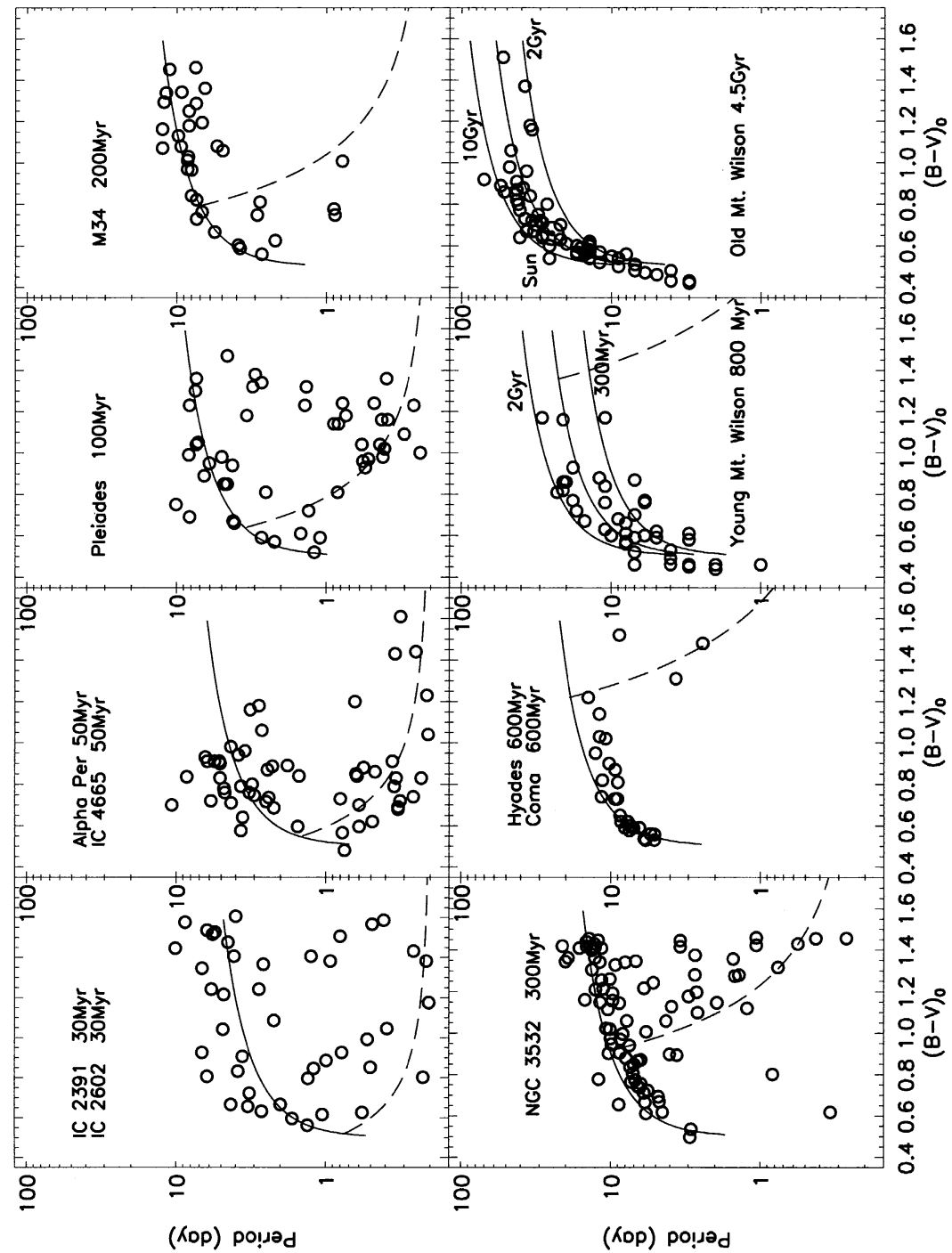

Figure 4. The rotation period observations in open clusters, augmented by the Mt. Wilson stars. The overplotted curves suggest the underlying sequences. Source: Barnes (2003). 
the evolution of the rotation rates. The myriad processes postulated to explain each separate feature of the evolution have produced a confusing and aesthetically untenable situation which required a careful re-examination of the growing amount of the most reliable data, namely, rotational periods of many stars in open clusters of different ages.

The results of the above re-examination suggest a unified picture of the problem that may constitute a new paradigm to study the problem. If we plot rotation rate vs. color, or any similar indication of mass, we find most stars to lie on one of two branches, with some stars found in the gap between them. It appears that stars are born on either branch, but that, as time goes on, those from the lower branch (the fast rotators, also denoted as saturated branch) move up to the upper (slow or interface) branch after a brief transition through the gap. At present, we have a broad physical picture of the internal dynamics of stars on either branch, and we can quantitatively compute their evolution over time. What we cannot currently predict is precisely when, and how, the individual transitions take place. This phenomenon is inexorably tangled with the difficult problem of stellar dynamos, which is still far from being solved, even for the Sun.

The above findings are exciting for a variety of reasons. Besides providing a framework for which otherwise difficult to comprehend data can be neatly classified, and formally understood, the most exciting prospect is its potential for addressing the stellar dynamo problem, and the effects of the rotational evolution in mixing of the material of the stellar interiors, and its effect on anomalous chemical abundances, particularly of trace elements.

Acknowledgments. This work was primarily supported by the NSF through AST-9986962. Additional NSF support was provided through ATM0206130 .

\section{References}

Barnes, S.A., 2001, ApJ 561, 1095

Barnes, S.A., 2003, ApJ 586, 464

Barnes, S.A. \& Sofia, S., 1996, ApJ 462, 746

Barnes, S.A., Sofia, S. \& Pinsonneault, M.H., 2001, ApJ 548, 1071

Chaboyer, B., Demarque, P. \& Pinsonneault, M., 1995, ApJ 441, 865

Choi, P.I. \& Herbst, W., 1996, AJ 111, 283

Donahue, R.A., 1998, in ASP Conf. Ser. 154, Tenth Cambridge Workshop on Cool Stars, Stellar Systems and the Sun, ed. R.A. Donahue and J.A. Bookbinder, 1235

Edwards, S. Strom. S. E., Herbst, W., Attridge, J., Michael Merill, K. , Probst, R. and Gatley, I., 1993, AJ 106, 372

Endal, A.S. \& Sofia, S., 1981, ApJ 243, 625

Kawaler, S.D., 1988, ApJ 333, 236

Keppens, R., MacGregor, K.B., \& Charbonneau, P., 1995, A\&A 294, 469

Kraft, R., 1967, ApJ 150, 551

Krishnamurthi, A. , Pinsonneault, M.H., Barnes, S., \& Sofia, S., 1997, ApJ 480, 303

MacGregor, K.B. \& Brenner, M., 1991, ApJ 376, 204 
Palla, F. \& Stahler, S., 1991, ApJ 375, 288

Pinsonneault, M. H., Kawaler, S. D., Sofia, S. and Demarque, P., 1989, ApJ 338, 424

Rebull, L.M., 2001, AJ, 121, 1676

Skumanich, A., 1972, ApJ, 171, 565

Soderblom, D.R., Duncan, D.K., \& Johnson, D.R.H., 1991, ApJ 375, 722

Stassun, K.G., Mathieu, R.D., Mazeh, T., \& Vrba, F.J., 1999, AJ 117, 2941

Stauffer, J.R., 1994, in ASP Conf. Ser., Vol. 64, Proc. 8th Cambridge Workshop on Cool Stars, Stellar Systems, and the Sun, ed. J.-P. Caillault (San Francisco: ASP), 163

Tinker, J., Pinsonneault, M. \& Terndrup, D.M., 2002, ApJ 564, 877

Van Leeuwen, F., \& Alphenaar, P., 1982, ESO Messenger 28, 15

Van Leeuwen, F., Alphenaar, P., \& Meys, J.J.M., 1987, A\&AS 67, 483

Vaughan, A.H., 1980, PASP, 92, 392

Wilson, O.C., 1963, ApJ 138, 832 